\title{
Fungal Mass in the Concha Bullosa: A Rare Entity
}

\author{
${ }^{1}$ Vivek Sasindran, ${ }^{2}$ Antony Joseph, ${ }^{3}$ Shobin Suja Abraham, ${ }^{4}$ Amol Gautam
}

\begin{abstract}
The enlargement of the middle concha as a pneumatized cavity is defined as concha bullosa. Concha bullosa is one of the most frequently encountered anatomic variations inside the nose. Polyps, submucous cysts, ossifying fibromas and pyoceles have been found in concha bullosa. This is a case report where fungal mass was seen inside the concha bullosa. Clinicians should be aware that a chronic rhinosinusitis that is unresponsive to normal management with a hyper dense focus is highly suggestive of fungal ball.
\end{abstract}

Keywords: Concha bullosa, Fungal ball, Aspergillus infections.

How to cite this article: Sasindran V, Joseph A, Abraham SS, Gautam A. Fungal Mass in the Concha Bullosa: A Rare Entity. Clin Rhinol An Int J 2014;7(3):132-134.

\section{Source of support: Nil}

Conflict of interest: None

\section{INTRODUCTION}

Fungal balls or mycetomas are extramucosal accumulations of degenerating fungal hyphae especially within chronically inflammed paranasal sinuses. Aspergillus infections of the nose and paranasal sinuses are being increasingly recognized in recent years. Fungal balls confined to the concha bullosa alone without involving the paranasal sinuses is a rare presentation and we have found only five reported cases in literature till date.

\section{CASE REPORT}

A 14-year-old girl presented to us with recurrent rhinitis, left sided nasal obstruction for the past 12 months. It was associated with headache, frontal heaviness and few episodes of epistaxis. Anterior rhinoscopy revealed deviation of the septum to the right side. There was a smooth surfaced, glistening, pinkish, globular mass

\footnotetext{
${ }^{1,4}$ Associate Professor, ${ }^{2}$ Professor and Head

${ }^{3}$ Postgraduate Resident

${ }^{1-4}$ Department of ENT, Pushpagiri Institute of Medical Sciences and Research Centre, Tiruvalla, Kerala, India
}

Corresponding Author: Vivek Sasindran, Associate Professor, Department of ENT, Pushpagiri Institute of Medical Sciences and Research Centre, Tiruvalla, Kerala, India, Phone: 9947132343, e-mail: shobinabraham0312@gmail.com with prominent blood vessels occupying the entire nostril just behind the anterior end of the inferior turbinate. On nasal endoscopy; the mass seemed to be arising from the middle turbinate. The middle turbinate was not visualized separately. It was insensitive to touch and did not bleed on probing. CT showed a well defined heterogeneously enhancing lesion in left middle turbinate wit bony fragments within it, causing mass effect, bony erosion and complete blockage of the left osteomeatal complex, with severe chronic left maxillary and ethmoid sinusitis; secondary to obstruction, as shown in Figure 1. Figure 2 shows a postcontrast axial cut CT. MRI revealed a mucosal thickening in the peripheral aspect of left concha bullosa with large T2 hypointense centre causing expansion of the concha bullosa (Fig. 3). The patient underwent endoscopic resection of the concha bullosa, during which a dark brown, cheese-like material was found. Histopathology reports confirmed the intraoperative findings.

On postoperative follow-up of this patient, she was relieved of her nasal symptoms. Endoscopic examination 1 month after surgery showed completely open bilateral nasal passages with no sign of any infection.

\section{DISCUSSION}

Concha bullosa occurs when the middle turbinate becomes pneumatized. This pneumatization results when ethmoid air cells migrate to the middle concha. As noted previously, this condition is a very common anatomic variation with a 14 to $53.6 \%$ reported frequency by various studies. ${ }^{1}$ The most common symptoms are nasal obstruction and facial pain. If the concha bullosa obstructs the middle meatus, the patient may develop sinusitis. ${ }^{2}$ The recent rise in mycotic nasal and paranasal infections is due to both improved diagnostic ability with wide use of computerized tomography in sinonasal pathologies and an increase of the conditions that favor fungal infections such as immunosuppressive diseases and neoplastic conditions. ${ }^{3}$ Fungal sinusitis has been classified as invasive and noninvasive forms, based on the presence or absence of hyphae in adjacent mucosa. Each group is subdivided into two subcategories, fulminant and chronic for the invasive form and fungal ball (previously called aspergilloma or mycetoma) and allergic for the noninvasive disease. ${ }^{4}$ In the head and neck region, Aspergillus species can cause otomycosis, 


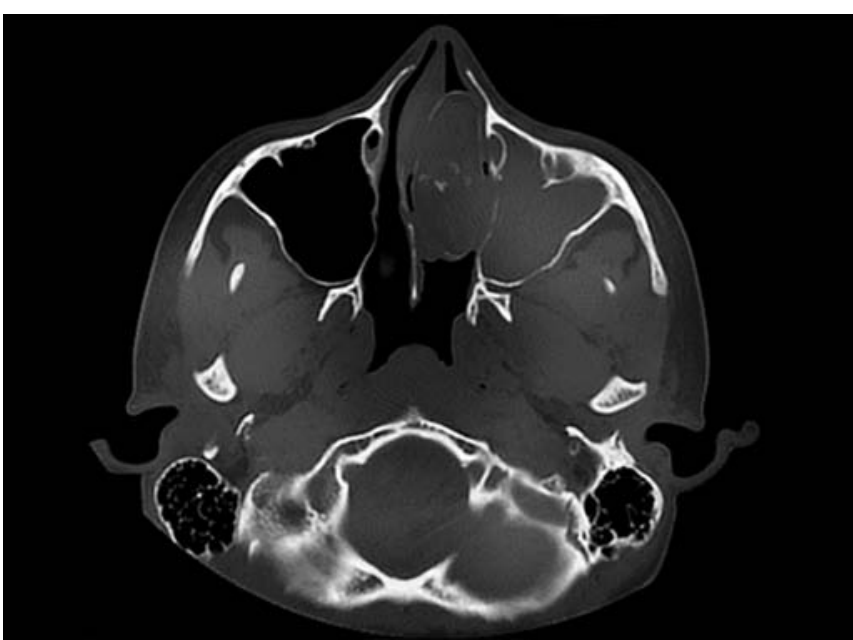

Fig. 1: Plain bone window, axial cut CT showing expansion of the concha bullosa abutting the medial wall of the maxillary sinus laterally and deviation of the septum

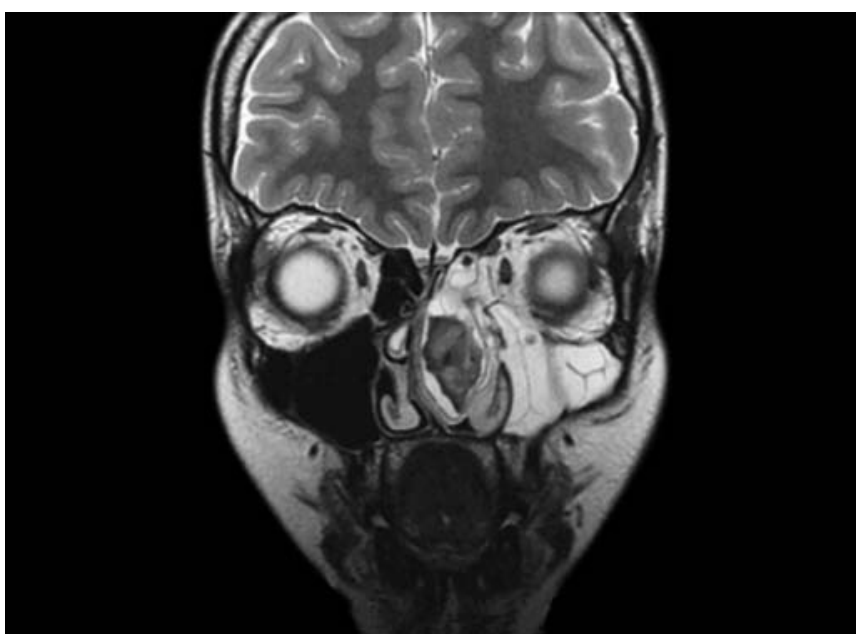

Fig. 3: Coronal T2-weighted MR image showing soft tissue in the concha with hypointense areas of signal void suggestive of calcifications

allergic paranasal sinusitis, invasive paranasal sinusitis, and aspergilloma of the paranasal sinuses. ${ }^{3,5}$ Paranasal fungal balls are noninvasive lesions with no evidence of spread into or beyond the sinus mucosa. The most common causative agents are Aspergillus sp., mainly A. fumigatus and $A$. flavus, less frequently. ${ }^{6}$ Cultures are often negative, and the fungus is identified in only 23 to $50 \%$ of cases, probably because of the low viability of the fungal components within the ball. Due to common appearance of Aspergillus sp., A. fungus ball is often mischaracterized as aspergillosis or aspergilloma. ${ }^{4-7}$ Conditions that favor fungal infections are diabetes, long-term treatments (antibiotics and steroids), radioand chemotherapy, immunosuppressive treatments, and immunodeficiency diseases. ${ }^{3}$ Many authors suggest that mycotic infections of the paranasal cavity are found more commonly in apparently healthy patients. From the imaging standpoint the radiological clues are apparent in all patients. Standard radiographs show unilateral

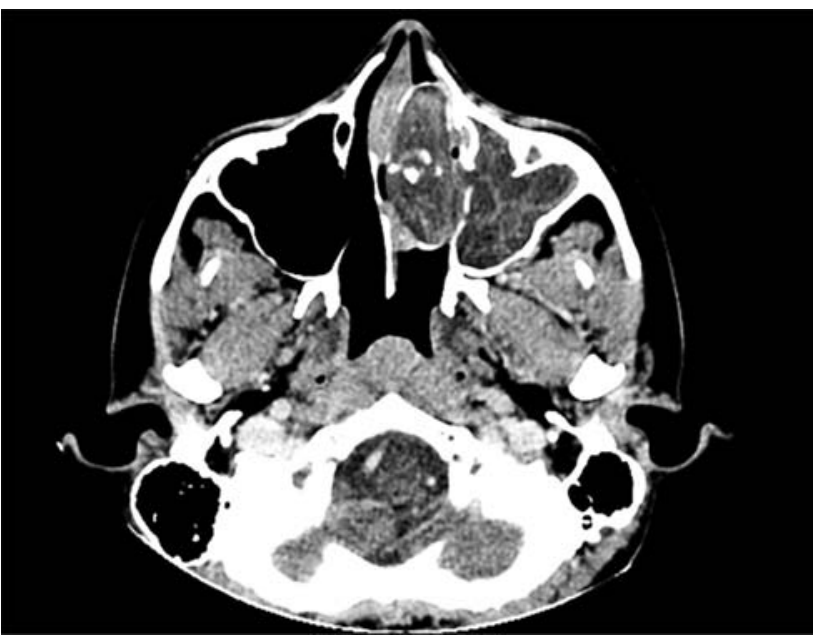

Fig. 2: Post contrast axial cut CT image showing soft tissue attenuated lesion within the concha causing expansion of the concha bullosa with areas of calcifications

partial or complete opacification of a sinus, most often the maxillary sinus. Characteristic CT scan findings include opacification with a hyperdensity in its core. Areas of hyperdense well-defined foci are observed in 25 to $50 \%$ of cases, strongly suggesting the diagnosis of a sinus fungus ball. Macroscopically, the fungus ball appears friable and grumous, may be green, yellow brown, or black, and detaches easily from the mucosa. Magnetic resonance imaging is often used for further evaluation of affected areas when aggressive surgical intervention is planned. Definitive diagnosis is based chiefly on the macroscopic appearance and histopathology, as cultures are frequently negative ( $70 \%$ of the cases). ${ }^{8}$

In a retrospective study done on various pathologic entities within the concha bullosa by Ibrahim Cukurova et al on 234 cases of concha bullosa, only $203(86.8 \%)$ of these were found to be a pneumatized cavity. The remaining 31 (13.2\%) cases had a pathologic entity within the concha bullosa. The cavity was filled with purulent material in 13 (5.6\%) of the cases, and bony septum (3\%), pyocele $(1.7 \%)$, ossifying fibroma $(0.4 \%)$, fungal ball $(0.4 \%)$, cholesteatoma $(0.4 \%)$ were other pathologic entities seen in the concha bullosa, in the study. ${ }^{9}$

Ciger E et al reported a similar case of a 29 -yearold woman who presented with nasal obstruction and postnasal discharge. Paranasal computed tomography demonstrated that the concha bullosa in the right middle turbinate was filled with a high density material. The patient underwent endoscopic resection of the concha bullosa, during which a dark brown, cheese-like material was found. Histopathological examination of the excised specimen revealed a fungus ball. ${ }^{10}$

In all the reported cases, computed tomogram and magnetic resonance imaging helped in arriving at a diagnosis which was confirmed intraoperatively 
by the macroscopic appearance of the fungal ball and postoperatively by the histopathological reports. Similarly in our patient, the fungal debris was confined to the concha bullosa, causing symptoms of sinusitis possibly secondary to obstruction by the enlarged concha bullosa; a rare presentation, which can be overlooked by the treating otorhinolaryngologists if not considered as a differential diagnoses.

\section{CONCLUSION}

Fungus balls are noninvasive accumulations of dense fungal concretions that generally affect healthy individuals. In the sinonasal region, they occur most frequently in the maxillary sinus. Although, fungus ball formation in the concha bullosa is unusual, it should be considered in the differential diagnoses of middle turbinate and sinonasal pathology. ${ }^{10}$

\section{REFERENCES}

1. Zinreich S, Albayram S, Benson M, Oliverio P. The ostiomeatal complex and functional endoscopic surgery. Head and Neck Imaging, P. Som, editor, pp. 149-173, Mosby, St Louis, Mo, USA, 4th edition, 2003.
2. Özkırış M, Karaçavuş S, Kapusuz Z, Saydam L. The impact of unilateral concha bullosa on mucociliary activity: an assessment by rhinoscintigraphy. Am J Rhinol Allergy 2013 Jan;27(1):54-57.

3. Costa F, Polini F, Zerman N, Robiony M, Toro C, Politi M. Surgical treatment of Aspergillus mycetomas of the maxillary sinus: review of the literature. Oral Surg Oral Med Oral Pathol Oral Radiol Endodontol 2007;103(6):e23-e29.

4. Grosjean, Weber R. Fungus balls of the paranasal sinuses: a review. European Archives of Otorhinolaryngology 2007; 264(5):461-470.

5. Scholar MK, Ozçelik BT, Saydam L, Kutluay L. A case of isolated aspergillosis of the maxillary sinus. Kulak Burun Boğaz İhtisas Dergisi 2008;18(1):53-55.

6. Durbec M, Bienvenu AL, Picot S, Dubreuil C, Cosmidis A, Tringali S. Maxillary sinus fungal infection by Acremonium. European Annals of Otorhinolaryngol, Head and Neck Diseases 2011;128(1):41-43.

7. Klossek E, Serrano L, Péloquin J, Percodani J, Fontanel P, Pessey JJ. Functional endoscopic sinus surgery and 109 mycetomas of paranasal sinuses. Laryngoscope 1997;107(1):112-117.

8. Ferguson BJ. Fungus balls of the paranasal sinuses. Otolaryngologic Clinics of North America 2000;33(2):389-398.

9. Cukurova I, Demirhan E, Karaman Y, Yigitbasi OG. Extraordinary pathologic entities within the concha bullosa. Saudi Med J 2009;30(7):937-941.

10. Ciger E, Demiray U, Onal K, Songu M. An unusual location for a fungus ball: the concha bullosa. J Laryngol Otol 2012 Aug;126(8):844-846. 